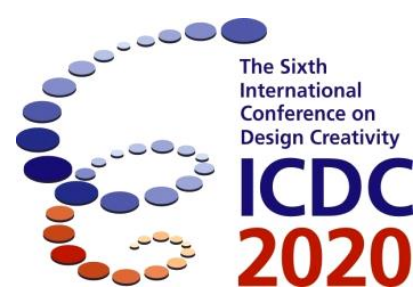

\title{
A Bottom-up Functional Domain Synthesis Approach for Creative Conceptual Design
}

\author{
Yutong Li and Yuxin Wang \\ Department of Mechanical and Electrical Engineering, \\ China University of Petroleum, East, Qingdao, China
}

\begin{abstract}
For expanding the solution space of conceptual design, a bottom-up functional synthesis approach through a partition of the functional domain of the design problem for mechanical products based on the equivalence relations in granularities, topologies, and attributes based on the domain synthetic theory in quotient space is proposed. The equivalence partition rules are objective and independent of a designer's experience and knowledge. Different equivalence partition rules will lead to different sub-functions, working principles, and function-structures to be generated. Then, the partition schemes based on the granularity equivalence relations are secondly divided into two quotient spaces corresponding to the time sequence equivalence relation and the space position equivalence relation, respectively. In this way, the synthesized sub-functions, which are formed and contributed by all of the basic operation actions in the divided subsets, can take place in time and space continuously and orderly.
\end{abstract}

Keywords: creative conceptual design, functional domain synthesis, time order, space order

\section{Introduction}

Making innovation is recognized as one of the most successful strategies for enterprises to capture market share, increase profit, and even survive (Hamel, 1998). Conceptual generation is the most critical stage of the design process that affects the creativity of the products. Creative design involves the production of new search spaces and the development of a variety of solutions (Stal \& Turkiyyah, 1996). Computer-based means of design synthesis enable designers to explore a broader range of creative solutions by eliminating a significant number of tedious design tasks. Chakrabarti et al. (2011) provided a detailed review of the advancements in computational design synthesis in the function-based, grammar-based (Schmidt \& Cagan, 1997), and analogy-based synthesis realms in the last decades and the significant challenges in further research. Under the premise of affirming that many talented researchers around the world have made great achievements, some hard challenges remain in the domain of computational function-based synthesis. For instance, explore large solution spaces created by design synthesis approaches with appropriate and effective search strategies. Obtain valuable and optimal conceptual design results. Implement the systematic design model presented by Pahl et al. (2007) with computational tools entirely. And especially, enhance computer-based functional synthesis systems to support problem-solving at the levels of complexity expected in practice.

The creativity of the outcome for conceptual design is influenced by the total number of searches occurring in each stage of the conceptual design. Sarkar \& Chakrabarti (2014) observed that searches occurring at the higher levels of the search hierarchy have a significant influence on the novelty of the 
outcomes. Chakrabarti \& Bligh (2001) pointed out that the solution-neutral functional description and the concept of functional primitives can help to improve the abstractness of the functional description and to expand the solution space of the conceptual design consequently. Through a detailed analysis of three main functional reasoning models, Erden et al. (2008) indicated that the performance of functional modelling with AI-based techniques depends on the modelling approach and the search space of the AI techniques employed. If the definition of functions is too precise, the search space will be restricted in a very narrow solution scope. For instance, the direct mapping from functions to structures in the FBS model leads to the application of AI techniques restricted in search of matching functions to structures. On the other hand, the majority of AI-based function reasoning processes apply top-down, one-way procedures starting from the requirements and moving toward their realization. Instead of a downward direction, Umeda \& Tomiyama (1997) proposed a decomposition procedure following a simultaneously top-down and bottom-up approach. Whereas the top-down process decomposes functions into subfunctions, a bottom-up process results in the recognition of high-level functions from the lower-level sub-functions. While the top-down and bottom-up functional decomposition or search strategies are applied in a functional reasoning process, the new search space, bottom-up functional synthesis, will be generated. In this way, the creativity of the outcomes of the conceptual design in the functional reasoning stage will be consequently enhanced. For enabling the function synthesis to tackle the creative design problems of non-routine and complex mechanical systems, Li et al. (2014) proposed a functionstructure bi-directional mapping schema. By introducing two bottom-up dynamic partitioning and regrouping processes between function layer and form layer, substituting the direct mapping process between functions and artifacts in the FBS model, the computerized model supporting non-routine conceptual design problems is presented. Continually, this paper proposes a functional domain synthesis approach for generating novel functional structure and novel functional schemes without the functional reasoning process.

\section{Behaviour segments}

\subsection{Behaviour segments}

The kinematic behaviours of mechanisms can be viewed as being constituted in sequence and order by several simple behaviours segments independent from mechanisms. Behaviours segments are part of complex mechanical systems' kinematic behaviours, like the linkage curve shown in Fig. 1a can be divided into three arcs, i.e., three behaviours segments. If the behaviours segment set is applied to represent the motion behaviours of the complex mechanical system, the creative conceptual design of the complex system based on the behaviours segment set can be realized with the aid of the function synthesis method. Through domain synthesis, i.e., division and reconstruction of the behaviours segment set in functional layer, the novel functional structure or functional principles can be generated. The solution space in functional layer is expanded consequently. Furthermore, the functional layer and the structure layer can match each other without needing a function reasoning process. In this way, the non-routine design problem for creative conceptual design can be treated. Behaviours segments, on the other hand, can depict not only constituent mechanisms' input-output behaviours and intermittent behaviours, etc., but also some specific kinematic behaviours, such as coupler curves. So it can enable the constructed conceptual design platform to solve the creative design problems of generally complex mechanical systems. Because the granularity of behaviour segments is smaller than the kinematic behaviours of basic mechanisms, especially, adopting behaviours segments to depict the kinematic behaviours of basic mechanisms can expand the solution space of conceptual design sufficiently, which improves the creativity of conceptual design.

\subsection{Characteristics and representation of behaviour segments}

The characteristics of behaviour segments are: they can represent behaviours and are the constituent units of complete behaviours. A single behaviour segment is independent of a mechanism, but a complete behaviour constituted of the behaviour segment sub-set needs to be implemented by constituent mechanisms in database. Behaviour segments have the features of the directivity, the continuity, and the reversibility. For instance, the coupler curve in Fig. 1a can be divided into three 
behaviour segments $C_{C r a c}$ (curve), $C_{C r c b}$ (curve) and $L_{b a}$ (straight line). The directivity (or order) means the behaviours occur in the order of $C_{C r a c} \rightarrow C_{C r c b} \rightarrow L_{b a}$; the continuity means these behaviours connect smoothly and continuously in the conjunctions $a, b, c$; and the reversibility means these behaviour segments can occur in the reverse sequence.

The mathematic descriptions of behaviours segments include: origins, attributes and kinematic features, as shown in Fig. 1b. The origins of one behaviour segment indicate which sub-function in function layer of FBS model it comes from, and which main function or secondary function of the constituent mechanisms it comes from. Behaviours attributes include kinematic patterns, such as rectilinear motion, smooth motion, intermittent, etc., behaviour types, behaviour features, and behaviour interface features.
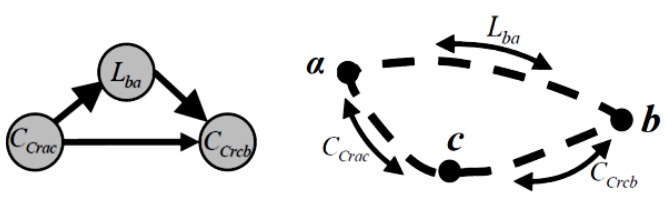

(a) A coupler curve

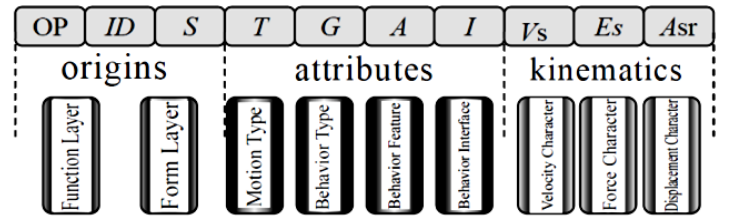

(b) Mathematic description

Figure 1. Description of a coupler curve with behaviours segments

\subsection{Basic operation action set}

In Fig. 1b, the mathematic descriptions of behaviour segments have contained the connect relations between the behaviours obtained from functional decomposition or constituent mechanisms' behaviour features, and the constraint conditions with other connected behaviour segments, as well as the interface conditions with which the behaviour occur. The segments in function layer and form layer are denoted with $b, b^{\prime}$ respectively, and their behaviours with $o p_{b}, o p_{s}$ :

$$
o p_{b}=\left\{o p_{b} \mid o p_{b} \in\left\{F_{o}, F_{c}, B_{v}, B_{c}, I_{c}, O_{c}, V_{b}, E_{b}, A_{b r}\right\}\right\}, \quad o p_{s}=\left\{o p_{s} \mid o p_{s} \in\left\{S_{o}, T_{s}, G_{m}, A_{i x}, I_{m}, O_{m}, V_{s}, E_{s}, A_{s r}\right\}\right\}
$$

Through analyzing the constituent behaviours' characteristics of commonly complex mechanical systems, the basic behaviours segment set of the constituent kinematic behaviours for general mechanical systems is constructed, and there are three categories, with total 284 items. Call the basic behaviours segment set constituting commonly complex mechanical systems as basic operation action set, and behaviours segments as basic operation actions (Li et. Al, 2014).

\section{Functional Domain Synthesis in Quotient Space}

\subsection{Description of basic operation actions}

The function decomposition process for mechanical systems has similar features with the hierarchical equivalence partition in quotient space (Zhang and Zhang, 2005). Therefore, the quotient space theory is used to descript the synthesis problems of basic operation actions in function layer. Let $(\mathrm{X}, \mathrm{f}, \mathrm{T})$ represent one basic operation action set. Here, $X=\{b 1, b 2, \ldots, b n\}$ is domain, i.e., the set of the basic operation actions contained in all the sub-functions in function layer; $\mathrm{T}$ is topological structure, describing the topological relations, including connecting relations, order relations, causal relations, condition relations and so on, which is used to determine the occurring sequence, temporal and special relations among basic operation actions. There exist order relation " $b i \rightarrow b j$ ”, parallel relation "bi $\Uparrow$ bj", or non-related relation "bi <> bj" between two basic operation actions. $\mathrm{f}$ is attribute function, describing basic operation actions' behaviour attributes, origin attributes and quality attributes as shown in Fig. 1, as well as basic operation actions' temporal attributes and special attributes.

Figure 2 shows the basic operation actions and their temporal and special relations for the function of a three-knife book trimmer, which are obtained with functional decomposition approach (Li et al., 2017). Table 1 demonstrates the temporal orders of these basic operation actions' antecedent nodes and succeeding nodes. These order nodes represent not only the connecting relations among the basic operation actions, but also the behaviour continuity relations. Both of them play an important role in behaviour clustering. Based on the defined spatial relation and temporal relation, these basic operation 
actions can be synthesized as continuous and ordered sub-functions behaviours, then implement the overall function synergistically.

\subsection{Topological structure synthesis of basic operation actions}

\subsubsection{Requirements for synthesis of kinematic behaviours of mechanical systems}

The synthesis of elements in $X=\left\{b_{1}, b_{2}, \ldots, b_{n}\right\}$ in the domain of quotient space is corresponding to a kind of partition of the domain. The synthesis of elements in is corresponding to a kind of partition of the domain. And each group of basic operation actions corresponds to synthesized behaviours. Such groups not only satisfy the condition of partition constraints, like granularity requirements but also satisfy behaviours' compatibility and consistency requirements, as well as the requirements that they can be mapped with the kinematic behaviours of existing mechanisms. According to set theory, one equivalence relation $R$ is corresponding to only one set partition. Thereby, the partition of domain $X$ can be implemented in quotient space based on the domain granularity, the equivalence of quotient attributes, and the equivalence of topological structure. The partition based on granularity is easier. Quotient attributes are in close relation to the kinematic characteristics of basic operation actions, and its synthesized results are sole. Generally, it needs to complement other conditions according to specific problems to obtain the attribute synthesis results. Therefore, this paper pays emphasis on introducing synthesis method of basic operation actions based on the equivalence partition of topological structure, including synthesis of temporal relations and spatial relations.

Table 1. Temporal and special relations among basic operation actions for the functions of the threeknife book trimmer

\begin{tabular}{|c|c|c|c|c|c|c|}
\hline \multirow{2}{*}{$\begin{array}{c}\text { Basic operation } \\
\text { actions }\end{array}$} & \multirow{2}{*}{$\begin{array}{c}\text { Behaviors } \\
\text { types }\end{array}$} & $\begin{array}{c}\text { Temporal attributes } \\
\text { Antecede } \\
\text { nodes }\end{array}$ & $\begin{array}{c}\text { Succeed } \\
\text { nodes }\end{array}$ & Origin & Terminal & Implementing \\
behaviors \\
\hline$b_{1}$ & $L$ & Non & $b_{3}$ & 0,1 & 3,1 & Deliver books \\
\hline$b_{2}$ & $L$ & $b_{3}$ & $b_{4}$ & 3,1 & 0,1 & Return \\
\hline$b_{3}$ & $L$ & $b_{1}$ & $b_{2}, b_{5}$ & 3,2 & 3,1 & Press books \\
\hline$b_{4}$ & $L$ & $b_{2}, b_{8}$ & Non & 3,1 & 3,2 & Backward \\
\hline$b_{5}$ & $L$ & $b_{3}$ & $b_{6}$ & 2,2 & 3,0 & Cut \\
\hline$b_{6}$ & $L$ & $b_{5}$ & $b_{7}$ & 3,0 & 2,2 & Return \\
\hline$b_{7}$ & $L$ & $b_{6}$ & $b_{8}$ & 4,2 & 4,0 & Cut \\
\hline$b_{8}$ & $L$ & $b_{7}$ & $b_{4}$ & 4,0 & 4,2 & Return \\
\hline
\end{tabular}

For mechanical systems, basic operation actions should occur in a certain order and sequence, thereby implementing the expected design functions synergistically. For instance, in the working process of the timing energy-saving automobile engine, the timing mechanism should guarantee to open or close the intake-exhaust valve when the piston operates at a particular location, to ensure the fuel-efficient burning in the engine. At the same time, when the system is running, kinematic behaviours are continuous in space, which means they cannot jump from one location to another. Thus, when partitioning the basic operation set, it should guarantee not only behaviours' continuity and sequence over time, but also the continuity in space. To obtain the partition schemes that satisfy not only temporal sequence relations but also spatial continuity, firstly, adopting temporal sequence partition $R_{t}$ to generate the quotient space $\left([X]_{t},[f]_{t},[T]_{t}\right)$, which satisfies the temporal sequence requirements. secondly, based on the requirement of spatial continuity, obtaining the quotient space, which satisfies the spatial equivalence relation $R_{p}$. Then, synthesizing the temporal quotient space and spatial quotient space $\left([X]_{p},[f]_{p},[T]_{p}\right)$, to get the combined quotient space $\left([X]_{c},[f]_{c},[T]_{c}\right)$, which satisfies both temporal sequence and spatial relations.

\subsubsection{Temporal sequence synthesis}

According to the temporal sequence requirements, the domain topology of the basic operation actions in mechanical systems, i.e. the topological connecting relation, belongs to semi-order space. The semiorder " $<$ " is defined as: 


$$
\forall b_{1}, b_{2} \in X, b_{1}<b_{2} \Leftrightarrow \text { basic operation action } b_{1} \text { happens before } b_{2} \text {. }
$$

The basic operation action set that satisfies the above definition is quotient semi-order space and can be represented with function directed network graphs, as shown in Fig. 2c. In this figure, each node represents an operation. And the arrow lines represent the semi-order between nodes, for instance, $b_{3} \rightarrow b_{5}$ represents that their order relation is $b_{3}<b_{5}$. Node $b_{1}$ is the antecedent node of node $b_{3}$, and node $b_{3}$ is the succeeding node of $b_{1}$.

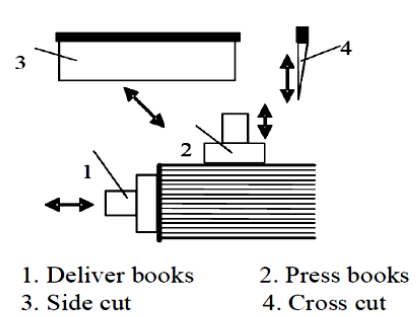

(a) Function requirements

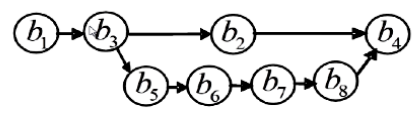

(c) Temporal relation

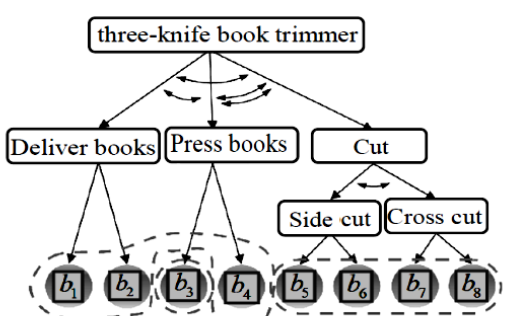

(b) Function-means tree

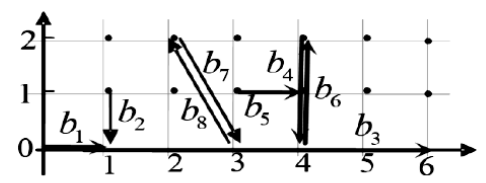

(d) Spatial relation

Figure 2. Basic operation actions and their relations for the functions of a three-knife book trimmer

It can also adopt temporal equivalence partition to obtain the synthesized space. To ensure the obtained function configuration schemes by partition satisfy the requirement of temporal order relations of basic operation actions in the function-means tree shown in Fig. 2, it needs to guarantee the elements in the obtained basic operation action sub-sets to satisfy the requirement of the temporal order relations as shown in Fig. 2c. Defining order-preserving equivalence relation $R_{S}=\left\{x_{n} \mid x_{n} \in X_{B}\right.$ and $\forall i, j \subset n$, there exist $\mathrm{t} x_{i}<x_{j}$ or $x_{i}>x_{j}$. The temporal order equivalence relation $R_{t}$ is the synthesis of granularity equivalence relation $R_{g}$ and order-preserving relation $R_{S}: R_{t}=R_{s} \wedge R_{g}$. Through the partition of the original basic operation space by equivalence relation $R_{t}$, the quotient space of temporal order $\left([X]_{t},[f]_{t},[T]_{t}\right)$ is obtained. Because the temporal parameters are variable, the partition based on temporal order relation can result in multiple partitions, which enlarges the function solution space.

For the function directed graph of the basic operation actions of a three-knife book trimmer, shown in Fig. 2c, one partition scheme that satisfies the temporal order relation in the graph is: $\left\{\left\{b_{1}, b_{3}, b_{2}, b_{5}\right\},\left\{b_{6}\right.\right.$, $\left.\left.b_{4}\right\},\left\{b_{7}, b_{8}\right\}\right\}$, as shown in Fig. 3a. The three groups of kinematic behaviours from the basic operation subsets satisfy the requirement of temporal order relation according to the function-means tree. Different partition rule $R_{t}$ can obtain different kinematic behaviours exhibited by the basic operation action sub-sets, i.e., different sub-functions, and different function-structures. Thus, the innovation of function principles in function layer is implemented. Obviously, the functional hierarchies based on equivalence partition can satisfy the overall functional requirement of design problems.

\subsubsection{Synthesis of spatial relation}

Different from the multiple solutions in temporal order relation partition, spatial relation partition needs to adopt the principle of maximum granularity, which groups all the basic operation actions that satisfy spatial continuity to one partition. The grouping principles are: 1) Group all the basic operation actions that have continuous trajectory; 2) Group all the basic operation actions that occur at the same node; 3) For simplicity of schemes, group all the basic operation actions that operate at the same position. In Fig. $2 \mathrm{~d}$, the basic operation actions satisfy the third grouping principle, so they should be grouped together. Spatial relation is in close relation to the behaviour characteristics and spatial orientations of the basic operation actions, so it needs to be analyzed according to specific basic operation actions. Define spatial equivalence relation as: 


$$
R_{p}=\left\{x_{n} \mid x_{n} \in X_{B^{\prime}} \text { and } \forall i, j \in n \text { there exist } x_{i} \cdot S_{n}=x_{j} \cdot E_{n} \text { or } x_{i} \cdot E_{n}=x_{j} \cdot S_{n}\right\} .
$$

Where $n$ is the index set. $x_{i} \cdot S_{n}$ and $x_{j} \cdot E_{n}$ represent starting points and terminal points of basic operation actions. Partitioning the basic operation space with equivalence relation $R_{p}$, the quotient space that satisfies spatial relations is obtained. Figure $3 \mathrm{~b}$ shows a partition that satisfies the spatial space of the basic operation actions shown in Fig. 2d: $\left.\left\{\left\{b_{1}, b_{3}, b_{2}\right\},\left\{b_{7}, b_{8}\right\}\right\},\left\{b_{4}, b_{5}, b_{6}\right\}\right\}$.

\subsubsection{Synthesis of temporal order and spatial relations}

Synthesizing the quotient space derived from the temporal order relation $\left([X]_{t},[f]_{t},[T]_{t}\right)$ and the quotient space from the spatial relation $\left([X]_{p},[f]_{p},[T]_{p}\right)$, the synthesized quotient space of both relations is obtained $\left([X]_{c},[f]_{c},[T]_{c}\right)$. The synthesized domain is:

$$
[X]_{c}=\left\{x_{i} \cap y_{j} \mid x_{i} \in[X]_{t}, y_{j} \in[X]_{p}\right\} ; i, j \in N
$$

As shown in Fig. 3, the process of synthesis is: select a division scheme of temporal relations, find the intersection between each group in the scheme and each group in the spatial relation quotient space, take the intersection element as a new group, and get all the groups contained in the composite quotient space in turn. As shown in Fig. 3c, a set of partition and synthesis schemes that conform to both temporal and spatial relationships in Fig. 2.

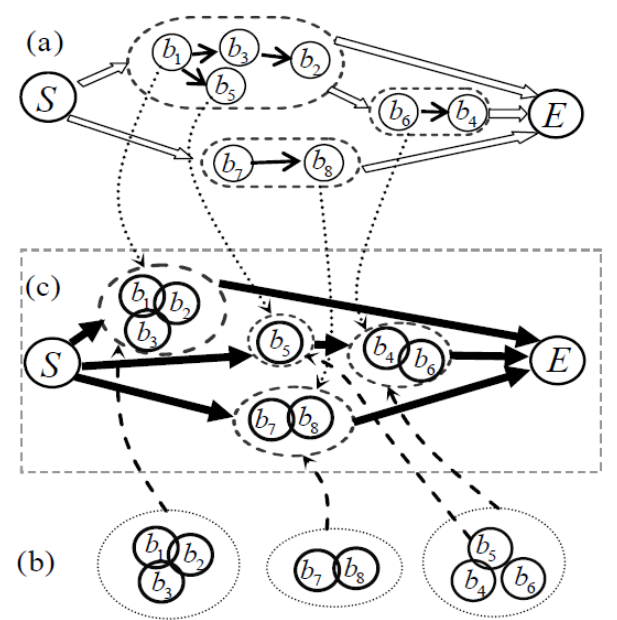

Figure 3. Synthesis of basic operation actions according to the order relations and the spatial relations

\section{Case study}

The function of locating and sheet transferring in printing machinery and printing post-processing machinery mainly includes the following sub-functions: transfer paper, press paper, locate, mount paper. After decomposing the overall function (Li, et al., 2017), the temporal order relations and spatial relations among the basic operation actions in function layer are obtained, as shown in Fig. 4a and Fig. 4b. Each basic operation action is as followings: b1 means the function of "transfer paper", whose behaviour is rectilinear motion from $[0,0]$ to $[1,0]$; b2 means the function of "press paper", whose behaviour is curve motion from $[1,2]$ to $[1,1]$; b3 means the function of "withdraw", whose behaviour is curve motion from $[1,1]$ to $[1,2]$; b4 means the function of "locate laterally", whose behaviour is rectilinear motion from $[3,2]$ to $[3,1]$; b5 means the function of "withdraw laterally", whose behaviour is rectilinear motion from $[3,1]$ to $[3,2]$; b6 means "locate horizontally", whose behaviour is curve motion from $[2,1]$ to $[1,1]$; b7 means withdraw horizontally", whose behaviour is curve motion from $[1,1]$ to $[2,1]$; b8 means the function of "fetch paper", whose behaviour is rectilinear motion from $[3,0]$ to $[4,0]$.

Set the grouping range $($ GMin, GMax $)=(1,4)$. The minimum and maximum number of basic operation actions included in the partition is $(\mathrm{OMin}, \mathrm{OMax})=(2,5)$, and the maximum group number of single operation is $(\operatorname{SoMax})=1$. According to the granularity equivalence relation: 


$$
R_{g}=\left\{R \mid \forall[x]_{\alpha} \in R_{i}, \exists[x]_{\beta} \in R_{j} \text { satisfies }[x]_{\alpha} \cdot l=[x]_{\beta} \cdot l\right\}
$$

where $[x]_{\alpha} \cdot l$ represents the number of the basic operation actions in group $[x]_{\alpha}$. Five partition schemes are obtained: $\{\{2\},\{3\},\{3\}\},\{\{3\},\{5\}\},\{\{4\},\{4\}\},\{\{2\},\{2\},\{4\}\},\{\{2\},\{2\},\{2\},\{2\}\}$. Among these schemes, there are in total 25 schemes that satisfy the order-preserving requirements. A part of these schemes are shown in Fig. 5.

Even though the partition schemes in the figure can satisfy the temporal order relations shown in Fig. $4 \mathrm{a}$, and can guarantee that the sub-behaviours constituted by the basic operation actions can occur in a certain order and continuously. There exist partition schemes whose spatial connections are unreasonable because the currently obtained partition schemes do not consider the spatial relations among the basic operation actions. As shown in Fig. 5a, the basic operation actions in the groups satisfy the temporal order relations, but because their spatial positions are not continuous, so they cannot be implemented by a single mechanism. To obtain the partition schemes that satisfy not only the requirement of the temporal order relations, but also the spatial continuity requirement, the intersection of the schemes meeting the temporal order relations and the schemes meeting the spatial relations can be synthesized. The four synthesized schemes that satisfy both the temporal order relations and the spatial relations in Fig. 4 are shown in Fig. 6.

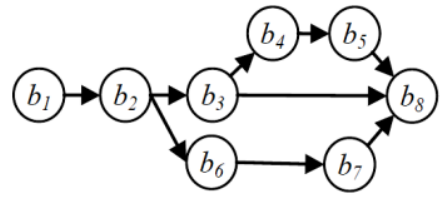

(a) Temporal order relations

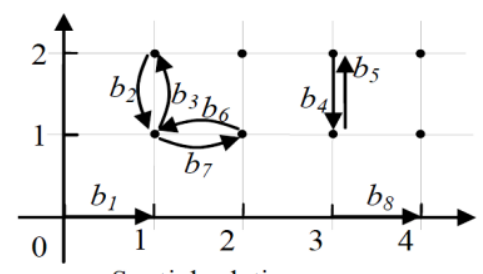

(b) Spatial relations among

Figure 4. The temporal order and/or spatial relations among the basic operation actions

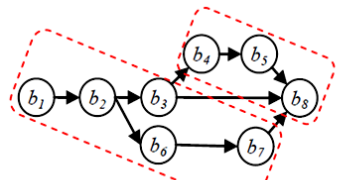

(a) $\{1,2,3,6,7\}\{4, \bar{s}, \dot{8}\}$

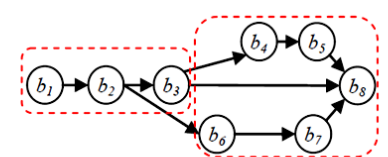

(b) $\{1,2,3\}\{4,5,8,7,6\}$

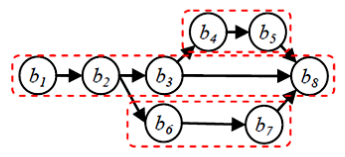

(c) $\{1,2,3,8\}\{4,5\}\{7,6\}$

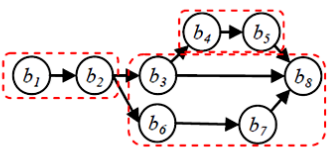

(d) $\{1,2\}\{3,8,4,5\}\{6,7\}$

Figure 5. The partition schemes that satisfy the temporal order relation

Here, Fig. 6a and Fig. 6b are the function behaviours similar with the compound front-guide paper transfer mechanism. Other synthesized schemes also satisfy the constraints of the temporal order relations and the spatial relations and can generate different design schemes through mapping artifacts, thus implementing innovation.

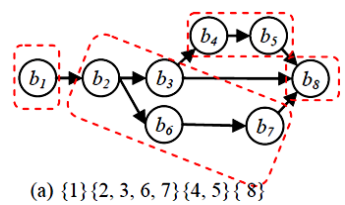

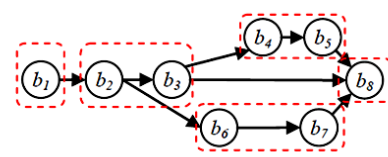

(b) $\{1\}\{2,3\}\{7,6\}\{4,5\}\{8\}$

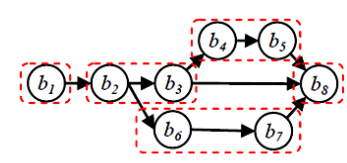

(c) $\{1\}\{2,3\}\{8\}\{4,5\}\{6,7\}$

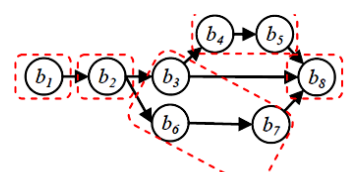

(d) $\left.\{1\}\{2\}\{3,7,6\}\{8\}\{4)^{-}-5\right\}$

Figure 6. The synthesized results of temporal order relation and spatial relation

\section{Discussion}

Similar to the proposed research by Umeda and Tomiyama (1997), the top-down and bottom-up functional synthesis processes are adopted in the proposed paper. Comparatively, the functional synthesis process in the former is a knowledge-based AI synthesis process, and the functional reasoning knowledge is necessary. However in the proposed research of this paper, the subfunctions in the FBS model are divided into the basic operation actions with finer granularity. Through regrouping of these basic operation actions in functional layer, new subfunctions and novel functional structures are 
generated. The purpose of the presented paper is for dealing with the regrouping of these basic operation actions so that the regrouped subfunctions can take place in time and space continuously and orderly to implement the overall function of the design problem. Since the regrouping process is rule-based, i.e., based on the granularity equivalence relations, the functional syntheses process does not need functional reasoning knowledge to support.

Two strategies for expanding solution space of conceptual design are adopted in the proposed paper. The first one is with finer granularity basic operation actions describing the subfunctions in functional layer. The second is through the top-down and bottom-up functional synthesis based on the equivalence partition rules to produce different function configuration schemes.

The advantage of the proposed method is that the function synthesis process does not need the function reasoning knowledge to support so that it can deal with non-routine innovative design problems. Due to using fine-grained basic operation actions to express subfunctions, the functional decomposition and synthesis solution space can be greatly expanded, and the proposed method has a strong ability to obtain innovative solutions.

As far as the research progress is concerned, the automation of innovative design based on the ideal hypothesis of basic operation actions set has been mainly solved. But for the innovative design of practical systems, such as the mechanical system, the practical basic operation actions set corresponding to the specialized domain knowledge should be established at first. This work is enormous but encourageable.

\section{Conclusion}

Based on the domain synthesis theory in quotient space, this paper presents a functional domain synthesis approach for generating novel functional structure and novel functional schemes without functional reasoning process. For making the synthetic subfunctions formed and contributed by all of basic operation actions in the divided subsets take place in time and space continuously and orderly, the partition schemes, which are generated based on the granularity equivalence relations, are secondly divided into two quotient spaces corresponding to the time sequence equivalence relation and the space position equivalence relation, respectively. The synthesized subfunctions corresponding to the intersection quotient space of these two quotient spaces can occur in time and space continuously and orderly. In this way, the functional domain synthesis approach proposed in this paper can not only generate novel functional principles and new functional schemes, but also guarantee the generated subfunctions take place in time and space continuously and orderly. These kinds of functional schemes will benefit from finding optimal structures to realize the overall function.

\section{Acknowledgement}

The authors gratefully acknowledge that this research project is supported by the NSFC with the grant number 51775557,51375496 and 50875238.

\section{References}

Hamel, G. (1998). Strategy innovation and the quest for value. Sloan Manage Review, 39(2): 7-14.

Stal, D. M., \& Turkiyyah, G. M. (1996). Skeleton-based techniques for the creative synthesis of structural shapes. Artificial Intelligence in Design'96, London: Springer-Verlag, 761-780.

Chakrabarti, A., Shea, K., Stone, R., Cagan, J., \& et al. (2011). Computer-based design synthesis research: an overview. J Com Infor Sci Eng, 11 (6): 021003-1-10.

Schmidt, L., \& Cagan, J. (1997). GGREADA: A Graph Grammar-Based Machine Design Algorithm. Res Eng Design, 9 (2): 195-213.

Pahl, G., Beitz, W., Feldhusen, J., \& Grote, K. H. (2007). Engineering design: a systematic approach, 3rd ed. London: Springer.

Sarkar, P., \& Chakrabarti, A. (2014). Ideas generated in conceptual design and their effects on creativity. Res Eng Design, 25(3): 185-201.

Chakrabarti, A., \& Bligh, T. P. (2001). A Scheme for Functional Reasoning in Conceptual Design. Des Stu, 22(6): 493-517.

Erden, M., Komoto, H., Beek, V., D’amelio, V., Echavarria, E., \& Tomiyama, T. (2008). A review of function modelling: Approaches and applications. Artif Intel Eng Des Anal Manu, 22(2): 147-169. 
Umeda, Y., \& Tomiyama, T. (1997). Functional Reasoning in Design. IEEE Int Sys App, 12(2): 42-48.

Li, Y. T., Wang, Y. X., \& Duffy, A. H. (2014). Computer-based creativity enhanced conceptual design model for non-routine design of mechanical systems. Chinese J Mech Eng (E), 27(6): 1083-1098

Li, Y. T., Wang, Y. X, \& MAO, X. H. (2017). Functional decomposition to computerized network diagram structure and implementation (In Chinese). Chinese J Mech Eng, 53(21): 128-135

Zhang, L. \& Zhang, B. (2005). A quotient space approximation model of multi-resolution signal analysis. J Com Sci Tech, 20(1): 90-94 